\title{
Gender-related Differences in the Obstructive Sleep Apnea Syndrome
}

\section{Geschlechtsspezifische Unterschiede beim Obstruktiven Schlafapnoesyndrom}

Author

Institution

\author{
A. Valipour
}

Department of Respiratory and Critical Care Medicine, Ludwig-Boltzmann-Institute for COPD and Respiratory Epidemiology, Otto-Wagner-Hospital, Vienna, Austria received $\quad$ 7.8.2012 accepted after revision 20. 8. 2012

Bibliography

DOI http://dx.doi.org/

10.1055/s-0032-1325664

Published online: 17.9.2012

Pneumologie 2012; 66: 584-588

(c) Georg Thieme Verlag KG

Stuttgart · New York

ISSN 0934-8387

Corresponding author

Arschang Valipour, MD, FCCP,

Assoc. Prof.

Department of Respiratory and

Critical Care Medicine,

Ludwig-Boltzmann-Institute

for COPD and Respiratory

Epidemiology, Otto-Wagner-

Hospital, Vienna, Austria

Sanatoriumsstraße 2

1140 Vienna

Austria

arschang.valipour@wienkav.at

\section{Abstract}

$\nabla$

The obstructive sleep apnea syndrome (OSAS) is associated with increased morbidity and mortality. Epidemiological studies suggest a two-fold higher prevalence of OSAS in men than in women. Differences in pharyngeal collapsibility and central respiratory drive may play a significant role in the gender-specific pathogenesis. Compared with their male counterparts, female patients experience OSAS at an older age (postmenopausal) and with higher body-mass-index. Female OSAS patients furthermore more frequently report atypical symptoms, such as insomnia, depression, and/or restless legs, and thus are less likely to be referred for the evaluation of sleep disordered breathing. Knowledge about these distinct gender-related differences in clinical features of OSAS may contribute to an increased awareness, improved diagnosis and its therapeutic consequences.

\section{Introduction}

The obstructive sleep apnea syndrome (OSAS), a disorder characterized by episodic collapse or narrowing of the upper airway during sleep, is an important cause of morbidity and mortality [1]. Closure of the upper airway results in hypoxemia which is accompanied by incremental breathing efforts that culminate in arousal, re-opening of the upper airway and brief hyperventilation followed by return to sleep. Patients suffering from this condition usually exhibit more than 15 apneic (complete flow limitation) or hypopneic (partial flow limitation) events during sleep. Repetitive arousals due to these events result in sleep fragmentation and increased sympathetic activity

\section{Zusammenfassung}

$\nabla$

Das obstruktive Schlafapnoesyndrom (OSAS) ist eine Erkrankung mit hoher Morbidität und Mortalität. Epidemiologischen Studien zufolge sind Männer doppelt so häufig von OSAS betroffen wie Frauen. Unterschiede bei pharyngealer Kollapsibilität und zentraler Atemregulation scheinen eine wesentliche Rolle in der geschlechterspezifischen Pathogenese zu spielen. Im Vergleich zu Männern mit OSAS tritt das Syndrom bei Frauen meist im späteren Lebensalter (post-menopausal) und bei höherem Body-Mass-Index auf. Frauen mit OSAS berichten häufiger über atypische Symptome, wie zum Beispiel Insomnie, Depression und/oder Restless Legs, und werden daher im Vergleich zu Männern seltener einer Abklärung zur Diagnostik von Schlafatemstörungen zugeführt. Das Wissen um die hier dargestellten geschlechterspezifischen Unterschiede in Klinik und Ausprägung des OSAS könnte zu einem erhöhten Bewusstsein für das Krankheitsbild bei Frauen und zu einer Verbesserung von Diagnostik und den entsprechenden therapeutischen Konsequenzen führen.

[2]. Sympathetic overactivity in combination with recurrent hypoxemia places OSAS patients at an increased risk of cardiovascular morbidity and mortality. Typical symptoms include snoring, excessive daytime sleepiness, and cognitive dysfunction. Gold standard of OSAS treatment involves continuous positive airway pressure (CPAP). CPAP alleviates symptoms of OSAS, improves quality of life, and reduces cardiovascular risk [3].

There are several known risk factors for OSAS such as obesity, increased age, cigarette smoking, and craniofacial abnormalities. Male gender is also recognized as an independent risk factor for the development of the syndrome. Epidemiological studies suggest a two to threefold higher prev- 


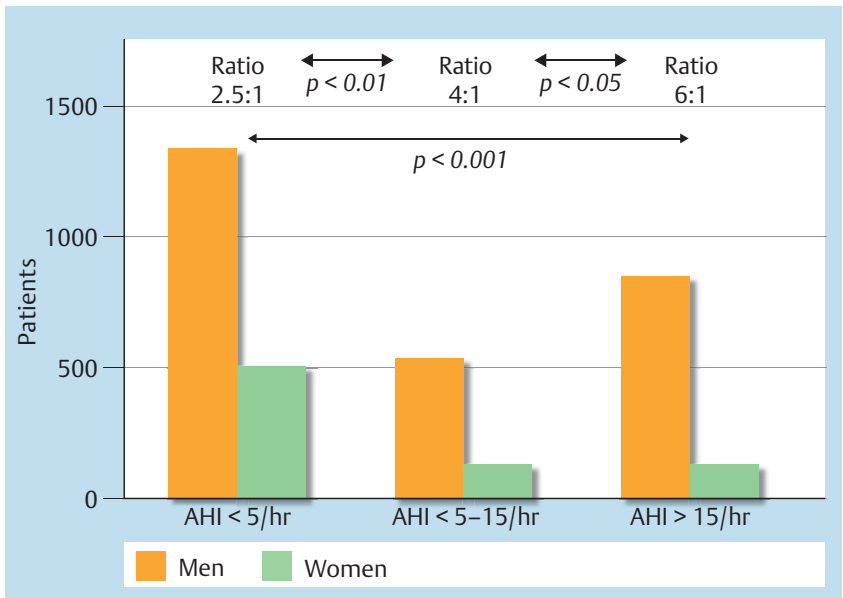

Fig. 1 Gender-related differences in Apnea-Hypopnea-Index in a large clinical population sample of patients who underwent overnight polysomnography (Valipour et al, Sleep 2007) [9].

alence of OSAS in men than women [4-6]. The ratio of men to women in the sleep laboratory sample, however, is higher than in the community and is typically 6:1 or greater ( $\mathbf{~ F i g . 1 ) ~ [ 7 ] . ~}$ Women who are diagnosed with the syndrome usually suffer from less severe sleep apnea, have a higher prevalence of apneic events during REM sleep, and are at average older than their male counterparts $[8,9](\circlearrowleft$ Tab. 1). Furthermore, compared with men, women have significantly higher health care consumption and report significantly lower perceived health status [10]. These factors suggest distinct gender-related differences in the mechanism, severity and clinical presentation of OSAS. The present article will review some of the evidence related to these genderspecific aspects in sleep apnea with particular emphasis on upper airway characteristics, the effects of sex-hormones, and the importance of gender-related differences in the clinical presentation of OSAS.

\section{The upper airway}

\section{$\nabla$}

A variety of reports investigated the influence of gender on upper airway characteristics and gender-related differences in upper airway collapse susceptibility. Guilleminault and co-workers, used cephalometric measurements in men and women with OSAS in order to demonstrate that women had shorter soft palates but equal posterior airway space when compared with men with similar sleep disordered breathing severity [11]. Another report studied upper airway dimensions in a large group of markedly obese male and female subjects using acoustic reflection technique [12]. Surprisingly, both the oropharyngeal junction and pharyngeal cross-sectional area were significantly larger in male than female patients. Rowley and co-workers similarly measured upper airway cross-sectional area in addition to retropalatal compliance in wakefulness and non-rapid eye movement sleep in equal number of men and women without sleep apnea [13]. Consistent with the previous observation, cross-sectional area tended to be larger in men compared with women in both wakefulness and sleep. After correction for body surface area, however, there were no significant differences in these measurements. Similar findings were observed for retropalatal compliance, which was higher in men during both wakefulness and
NREM sleep, but similar in men relative to women after correction for neck circumference. Whittle and co-workers used MRI studies to investigate gender-related differences in fat deposition in the regions prone to collapse during sleep [14]. The authors found that the pattern of fat deposition in the neck close to the upper airway differed between men and women, however, it appeared that the larger soft tissue mass in the male neck was mainly due to soft tissue other than fat. The major anatomical structures in the anterior segments inside the mandible at the palatal level, which showed greater fat deposition in men, are the soft palate and the upper part of the tongue. When analysing the cross sectional areas of tissue and fat in these structures in the medial sagittal plane, the authors found no sex difference in fat deposition [14].

On the basis of the above mentioned reports of a larger pharynx in men, one would predict that men have a lower predisposition of upper airway collapse than women, but the opposite is true. In fact, there is a relationship between pharyngeal size and severity of sleep apnea in men but not in women, indicating that OSAS in women is less associated with pharyngeal anatomic size rather than other factors, such as alterations in upper airway tone and/ or tissue elasticity [12].

Upper airway tone is mainly driven by upper airway dilator muscle activity, which is higher in patients with OSAS than in normal subjects during wakefulness [15]. During sleep the loss of muscle activation results in increased pharyngeal resistance and upper airway collapse [16]. Few studies with inconsistent results have examined the influence of gender on upper airway tone, some during wakefulness only, others during sleep, and few during wake to sleep transition. White and colleagues demonstrated higher pharyngeal resistance during wakefulness in healthy men in one study but similar waking pharyngeal airway resistance between men and women in another report $[17,18]$. During the wake-to-sleep transition, Thurnheer et al. observed no significant gender-related difference in upper airway resistance [19]. Trinder and co-workers extended these findings as they have similarly observed no gender-related differences in upper airway resistance during sleep transition, however, significantly higher upper airway resistance in men during NREM sleep, thus suggesting that the male pharyngeal airway is considerably more collapsible than the female pharynx during established sleep. [20]. Finally, Pillar and co-workers have shown that the greater increase in pharyngeal resistance in men was not due to genderrelated differences in central respiratory drive [21]. As most of the literature that examined gender-related differences in upper airway pathophysiology was conducted in subjects without sleep apnea, it remains difficult to draw definite conclusions on the effect of gender on upper airway tone in patients with OSAS.

\section{The role of hormones}

$\nabla$

Gender-related differences in the prevalence of OSAS decrease as age increases. The latter phenomenon is largely due to a significant increase in the prevalence of sleep disordered breathing after menopause [22]. In one of the earlier studies, Block and coworkers investigated nocturnal breathing in pre- and postmenopausal women. The authors showed a significantly higher prevalence of apneas, hypopneas, and episodes of oxygen desaturation in postmenopausal women [23]. Dancey and co-workers extended these findings as they have demonstrated that both prevalence and severity of sleep apnea increased in women after 
menopause, even after controlling for BMI and neck circumference [24]. The definition of menopause in both studies, however, was not based on objective measures of menopausal status.

In the Wisconsin Sleep Cohort, Young et al. used cross-sectional and longitudinal data on menstrual history, hormonal replacement therapy use, gynecologic surgery, and serum follicle-stimulating hormone levels to classify women on stage of menopause and years of postmenopause [22]. Menopausal transition in that report was associated with an increased likelihood of sleep disordered breathing irrespective of the AHI cut-off after controlling for age, body, habitus, and several lifestyle factors. Compared with premenopausal women, postmenopausal women in that report were 3.5 times more likely to have OSAH.

The overall lower prevalence of sleep apnea in women before menopause suggests a protective effect of female sex hormones on upper airway patency and/or ventilatory drive. In fact, Popovic and co-workers observed that the activity of the genioglossus muscle during wakefulness was lower in postmenopausal women than in premenopausal women, yet significantly increased in eight postmenopausal women after 2-week treatment with conventional doses of replacement hormones [25]. Vice versa, testosterone levels have been shown to be associated with upper airway collapsibility in patients with sleep apnea and there have been reports of sleep apnea in women by exogenous administration of testosterone [26,27]. These findings suggest sex hormone dependence of upper-airway dilator muscle activity. Progesterone and possibly estrogen may also play a role in the regulation of the ventilatory drive, raising the hypoxic ventilatory response and stimulating ventilation in conditions of hypoventilation [28, 29]. Declining levels of these hormones might therefore predispose some women to sleep-disordered breathing by lowering the ventilatory drive to the upper airway, which could lead to an imbalance between forces that compromise upper airway patency and counteracting forces, however, much more study is required to understand the complex relationship between sleep apnea and hormonal function.

On the basis of the above mentioned effects of sex-hormones, one might speculate that replacement therapy with female hormones may alleviate sleep disordered breathing. Studies that systematically investigated the effects of hormone replacement therapy in women have provided inconsistent results. While some reports have shown that administration of estradiol, estrogen, or progestin to postmenopausal women resulted in a reduction of time awake, an increase in the proportion of rapid eye movement sleep, and a reduction in apnea-hypopnea-index [30,31], others found no reduction in sleep-disordered breathing events after hormone replacement therapy in a group of postmenopausal women [32,33]. Most of these studies, however, suffered from small sample size and other methodological limitations.

Larger studies such as the one by Bixler et al. confirmed that postmenopausal women with hormone replacement therapy had a prevalence of sleep apnea that was similar to the prevalence in premenopausal women [34]. Vice versa, the absence of hormone therapy in postmenopausal women appeared to be associated with a higher prevalence of sleep apnea than that in premenopausal women. Further, the prevalence of sleep apnea in postmenopausal women without hormone replacement therapy was higher than in postmenopausal women with. Noteworthy, the prevalence of sleep apnea in postmenopausal women in that report remained significantly less than that in men, when controlling for age and body-mass-index.

\section{Polysomnographic features \\ $\nabla$}

One of the largest reports of polysomnographic features of OSAS was performed by O'Connor and co-workers [8]. In a retrospective study of 830 patients with OSAS, diagnosed by overnight polysomnography, the authors observed that the severity of sleep apnea was similar in women and men during REM sleep, but less severe in women during NREM sleep. Thus, women with OSA had a greater proportion of their respiratory events during REM sleep than do men, and a higher prevalence of REM related sleep apnea events (62\% REM-OSAS in women vs. $24 \%$ in men). In contrast, postural OSAS appeared to occur predominantly in men. These differences did not appear to be age- or weight dependent according to that report. Similarly, there was no evidence of an influence of medications, such as anti-depressants, on the prevalence of REM-OSAS. While these observations are consistent with the above stated sex-related differences in upper airway mechanics, the clinical relevance of these observations has yet to be determined. The long-term effects of (isolated or predominant) disruption of REM sleep, may contribute to explain why women are symptomatic at lower apnea-hypopnea-index compared with their male counterparts.

\section{Symptoms of OSAS in men and women \\ $\nabla$}

Despite increasing knowledge about the mechanisms that may contribute to explain why male gender is associated with a higher risk of developing sleep apnea, OSAS in women remains frequently underdiagnosed [35]. A variety of reasons may be responsible for the apparent gender discrepancy between clinic and community populations. It may be possible that fewer women seek help for sleep apnea symptoms than men. However, general care seeking patterns do not support the hypothesis [36]. In fact, Smith and co-workers demonstrated that women had significantly more frequent physician visits and hospitalizations prior to the diagnosis of OSAS than men [37]. The discrepancy between greater health care utilization and clinical underrecognition of OSAS in women may suggest that medical care providers fail to suspect or to diagnose sleep apnea in female patients. Using data from the Wisconsin University Sleep Laboratory, Young et al observed that nearly all men and women with an apnea-hypopnea-index greater than $20 / \mathrm{hr}$ were diagnosed with OSAS, but a lower proportion of women than men with an AHI in the range of $5-20 / \mathrm{hr}$ were given a diagnosis of sleep apnea [38]. This phenomenon may be due to gender-related differences in symptom expression at lower apnea-hypopnea-index particularly with respect to characteristic symptoms that have become the hallmark of the syndrome such as excessive daytime sleepiness, habitual snoring, and witnessed apneas.

Only few population-based studies systematically investigated gender-related differences in sleep disordered breathing symptoms. In the Wisconsin Sleep Cohort Study, there were no significant gender-related differences in snoring, self-reported breathing pauses, or restless legs in subjects with an AHI of $15 / \mathrm{hr}$ or greater [39]. It should be acknowledged, however, that this study may have been underpowered to detect a gender-specific difference in these symptoms, since only 17 female subjects with an AHI greater than $15 / \mathrm{hr}$ were included. In contrast, Redline and co-workers noted that the "classic" symptoms of sleep apnea, such as snoring and witnessed apnea were reported two- to three-fold more likely in males [4]. The best available evidence 
Table 1 Characteristics of OSAS in female patients.

Older age (frequently post-menopausal)
Higher body-mass-index
Lower AHI
REM-related OSAS
Atypical OSAS symptoms (Insomnia, Depression, Fatigue, Restless Legs)
Lower CPAP pressure requirements

from the community sample, however, comes from the Sleep Heart Health Study. Baldwin and colleagues demonstrated that women were significantly more likely to report insomnia, difficulties falling asleep, and awakening with leg cramps than men [40].

Studies conducted in the sleep laboratory have demonstrated a similar picture. In a retrospective chart-review analysis, Shepertycky and co-workers reported that women with OSAS less often reported on witnessed apnea compared with men [41]. Interestingly, women in that report described their main presenting symptoms as insomnia and were more likely to have a history of depression. Supporting these observations, we have similarly reported that women with suspected sleep disordered breathing were more likely to suffer from "atypical" symptoms, such as insomnia, depression, restless legs and nightmares [9]. In fact, an analysis of gender-related differences in morbidity and total health care utilization 5 years prior to diagnosis of OSAHS by Greenberg-Dotan and co-workers, demonstrated that women were significantly more likely to receive treatment with antipsychotics, anxiolytics, and/or antidepressants, suggesting that these patients may experience insomnia, anxiety, and depression [10].

Consistent with previous studies we also observed substantial gender-related differences in excessive daytime sleepiness in OSAS, with sleepiness being more pronounced in female patients. Curiously, in our clinical sample men had a significantly higher arousal index than women irrespective of AHI category. These observations may either suggest gender-related differences in the perception of sleepiness or a discrepancy between polysomnographic parameters of sleep fragmentation and subjective sleepiness reports. In fact, Kingshott and co-workers found no significant relationship between arousal frequency and subjective (Epworth Sleepiness Scale) or objective measures of sleepiness [43]. It has to be noted, however, that there is a strong relationship between excessive daytime sleepiness and complaints such as fatigue, depression or insomnia, symptoms which are also more commonly reported by women.

The findings from both clinical and population-based studies suggest that women suffer from a number of atypical symptoms of sleep apnea. This observation raised concerns as to whether frequently used diagnostic tools, such as the Epworth Sleepiness Score, may not be adequate enough to assess the full spectrum of OSAS symptoms in women. The distinction between the more traditional symptoms usually attributable to OSAS and the above-mentioned "atypical" symptoms is important, as clinicians rely more on self-reported apnea and the quantitative aspects of snoring, which have been associated with OSAS in predominantly male populations. The presentation of women with these atypical symptoms could make physicians turn to other diagnostic possibilities such as depression or insomnia, rather than to the actual diagnosis of sleep apnea. The latter may also explain greater utilization of health-care resources in women with OSAS compared with men [10].
The prevalence of OSAS in the general population is higher in men than women, yet the reasons for this gender disparity remains unclear. While there is some evidence suggesting increased susceptibility of the male pharynx to collapse during sleep, most of the studies that investigated the effect of gender on upper airway resistance were conducted in small samples of subjects without sleep apnea. Similarly, there is few data with respect to the effects of sex hormones on upper airway patency, which may explain some of the changes in OSAS prevalence and sleep apnea severity in females that occur after menopause. Given the evidence of clinical under-recognition of OSAS in women, a better understanding of the effect of gender-specific mechanisms and increasing awareness of the clinical presentation of OSAHS in women should be demanded both in the primary care as well as by sleep specialists. Finally, there appear to be gender-related differences in CPAP pressure requirements.

\section{Therapy}

There have been no systematic studies of gender-related differences with respect to the efficacy of non-CPAP therapies for OSAS.Similarly, there is paucity of data for the role of gender on CPAP acceptance, adherence, and/or efficacy. There appears to be no sex-difference in the type of interfaces used for CPAP therapy or in overall satisfaction with mask treatment [43]. A cross-sectional Japanese case-control study, however, observed that female patients require significantly lower levels of CPAP than male patients matched for BMI [44]. A more recent US study confirmed these findings and the effect of gender persisted after correction for the severity of OSA [45]. This observation is in line with evidence of a lower pharyngeal critical closing pressure in women. There are conflicting results as to whether there are gender-related differences in CPAP treatment adherence. Sin and coworkers [46] observed higher CPAP usage in female OSAS patients, a recent study in contrast observed slightly higher CPAP adherence data in male counterparts [47]. Unpublished data from our own institution is in line with the findings from Sin et al., with evidence of more regular usage of the CPAP device in female OSAS patients, which is consistent with higher usage of healthcare resources in female patients previously discussed [37]. While the magnitude of improvement in clinical outcomes does not vary by gender [48], Mermigkis and co-workers [49] recently demonstrated that CPAP therapy for OSAS may reverse elevated markers of systemic inflammation faster in male than female patients. This may suggest gender-related differences in CPAP effects on cardiovascular risk factors, however, further studies on the role of gender-related outcomes in response to OSAS treatment are required.

\section{Conflict of interest}

\section{$\nabla$}

The author has no conflict of interest. 


\section{References}

1 Marin JM, Carrizo SJ, Vicente E et al. Long-term cardiovascular outcomes in men with obstructive sleep apnoea-hypopnoea with or without treatment with continuous positive airway pressure: an observational study. Lancet 2005; 365: 1046-1053

2 Somers VK, Dyken ME, Clary MP et al. Sympathetic neural mechanisms in obstructive sleep apnea. J Clin Invest 1995; 96: 1897-1904

3 Maser RE, Lenhard MJ, Rizzo AA et al. Continuous positive airway pressure therapy improves cardiovascular autonomic function for persons with sleep-disordered breathing. Chest 2008; 133: 86-91

4 Redline S, Kump K, Tishler P et al. Gender differences in sleep disordered breathing in a community-based sample. Am J Respir Crit Care Med 1994; 149: $722-726$

5 Young T, Palta M, Dempsey J et al. The occurrence of sleep-disordered breathing among middle-aged adults. N Engl J Med 1993; 328: $1230-1235$

6 Duran J, Esnaola S, Rubio $R$ et al. Obstructive sleep apnea- hypopnea and related clinical features in a population-based sample of subjects aged 30 to 70 yr. Am J Respir Crit Care Med 2001; 163: 685-689

7 Kapsimalis F, Kryger MH. Gender and obstructive sleep apnea syndrome, Part 1: clinical features. Sleep 2002; 25: 412-419

8 O'Connor C, Thornley KS, Hanly PJ. Gender differences in the polysomnographic features of obstructive sleep apnea. Am J Respir Crit Care Med 2000; 161: 1465-1472

9 Valipour A, Lothaller H, Rauscher $H$ et al. Gender-related differences in symptoms of patients with suspected breathing disorders in sleep: a clinical population study using the sleep disorders questionnaire. Sleep 2007; 30: 312 - 319

10 Greenberg-Dotan S, Reuveni H, Simon-Tuval T et al. Gender differences in morbidity and health care utilization among adult obstructive sleep apnea patients. Sleep 2007; 30: $1173-1180$

11 Guilleminault C, Quera-Salva MA, Partinen $M$ et al. Women and the obstructive sleep apnea syndrome. Chest 1988; 93: 104-109

12 Mohsenin V. Gender differences in the expression of sleep-disordered breathing: role of upper airway dimensions. Chest 2001; 120: 1442 1447

13 Rowley JA, Sanders CS, Zahn BR et al. Gender differences in upper airway compliance during NREM sleep: role of neck circumference. J Appl Physiol 2002; 92: 2535-2541

14 Whittle AT, Marshall I, Mortimore IL et al. Neck soft tissue and fat distribution: comparison between normal men and women by magnetic resonance imaging. Thorax 1999; 54: 323-328

15 Mezzanotte WS, Tangel DJ, White DP. Waking genioglossal electromyogram in sleep apnea patients versus normal controls (a neuromuscular compensatory mechanism). J Clin Invest 1992; 89: 1571-1579

16 Mezzanotte WS, Tangel DJ, White DP. Influence of sleep onset on upperairway muscle activity in apnea patients versus normal controls. Am J Respir Crit Care Med 1996; 153: 1880-1887

17 White DP, Lombard RM, Cadieux RJ et al. Pharyngeal resistance in normal humans: influence of gender, age, and obesity. J Appl Physiol 1985; 58: 365-371

18 Popovic RM, White DP. Influence of gender on waking genioglossal electromyogram and upper airway resistance. Am J Respir Crit Care Med 1995; 152: $725-731$

19 Thurnheer R, Wraith PK, Douglas NJ. Influence of age and gender on upper airway resistance in NREM and REM sleep. J Appl Physiol 2001; 90: $981-988$

20 Trinder J, Kay A, Kleiman J et al. Gender differences in airway resistance during sleep. J Appl Physiol 1997; 83: 1986-1997

21 Pillar G, Malhotra A, Fogel $R$ et al. Airway mechanics and ventilation in response to resistive loading during sleep: influence of gender. Am J Respir Crit Care Med 2000; 162: 1627-1632

22 Young T, Finn L, Austin D et al. Menopausal status and sleep-disordered breathing in the Wisconsin Sleep Cohort Study. Am J Respir Crit Care Med 2003; 167: 1181 - 1185

23 Block AJ, Wynne JW, Boysen PG. Sleep-disordered breathing and nocturnal oxygen desaturation in postmenopausal women. Am J Med 1980; 69: $75-79$

24 Dancey DR, Hanly PJ, Soong $C$ et al. Impact of menopause on the prevalence and severity of sleep apnea. Chest 2001; 120: 151 - 155
25 Popovic RM, White DP. Upper airway muscle activity in normal women: influence of hormonal status. J Appl Physiol 1998; 84: 1055-1062

26 Cistulli PA, Grunstein RR, Sullivan CE. Effect of testosterone administration on upper airway collapsibility during sleep. Am J Respir Crit Care Med 1994; 149: 530-532

27 Johnson MW, Anch AM, Remmers JE. Induction of the obstructive sleep apnea syndrome in a woman by exogenous androgen administration. Am Rev Respir Dis 1984; 129: 1023-1025

28 Regensteiner JG, Woodard WD, Hagerman DD et al. Combined effects of female hormones and metabolic rate on ventilatory drives in women. J Appl Physiol 1989; 66: 808 -813

29 Robinson RW, Zwillich CW. The effects of drugs on breathing during sleep. Clin Chest Med 1985; 6: 603-614

30 Pickett CK, Regensteiner JG, Woodard WD et al. Progestin and estrogen reduce sleep-disordered breathing in postmenopausal women. J Appl Physiol 1989; 66: 1656 - 1661

31 Keefe DL, Watson R, Naftolin F. Hormone replacement therapy may alleviate sleep apnea in menopausal women: a pilot study. Menopause 1999; 6: 196-200

32 Cistulli PA, Barnes DJ, Grunstein RR et al. Effect of short-term hormone replacement in the treatment of obstructive sleep apnoea in postmenopausal women. Thorax 1994; 49: 699-702

33 Block AJ, Wynne JW, Boysen PG et al. Menopause, medroxyprogesterone and breathing during sleep. Am J Med 1981; 70: 506-510

34 Bixler EO, Vgontzas AN, Lin HM et al. Prevalence of sleep-disordered breathing in women: effects of gender. Am J Respir Crit Care Med 2001; 163: 608-613

35 Young T, Evans L, Finn L et al. Estimation of the clinically diagnosed proportion of sleep apnea syndrome in middle-aged men and women. Sleep 1997; 20: $705-706$

36 Cleary PD, Mechanic D, Greenley JR. Sex differences in medical care utilization: an empirical investigation. J Health Soc Behav 1982; 23: 106 119

37 Smith $R$, Ronald J, Delaive $K$ et al. What are obstructive sleep apnea patients being treated for prior to this diagnosis? Chest 2002; 121: 164 172

38 Young T, Finn L. Epidemiological insights into the public health burden of sleep disordered breathing: sex differences in survival among sleep clinic patients. Thorax 1998; 53: S16-S19

39 Young T, Hutton R, Finn $L$ et al. The gender bias in sleep apnea diagnosis. Are women missed because they have different symptoms? Arch Intern Med 1996; 156: 2445-2451

40 Baldwin CM, Kapur VK, Holberg CJ et al. Associations between gender and measures of daytime somnolence in the Sleep Heart Health Study. Sleep 2004; 27: 305-311

41 Shepertycky MR, Banno K, Kryger M. Differences between men and women in the clinical presentation of patients diagnosed with obstructive sleep apnea syndrome. Sleep 2005; 28: 309-314

42 Kingshott RN, Engleman HM, Deary IJ et al. Does arousal frequency predict daytime function? Eur Respir J 1998; 12: 1264 - 1270

43 Bachour A, Vitikainen P, Virkkula $P$ et al. CPAP interface: satisfaction and side effects. Sleep Breath. Epub ahead of print: 05. 07. 2012

44 Yukawa K, Inoue Y, Yagyu $H$ et al. Gender differences in the clinical characteristics among Japanese patients with obstructive sleep apnea syndrome. Chest 2009; 135: 337-343

45 Jayaraman $G$, Majid $H$, Surani S et al. Influence of gender on continuous positive airway pressure requirements in patients with obstructive sleep apnea syndrome. Sleep Breath 2012; 15: 781 - 784

46 Sin DD, Mayers I, Man GCW et al. Long-term compliance rates to continuous positive airway pressure in obstructive sleep apnea: A population based study. Chest 2002; 121: 430-435

47 Woehrle H, Graml A, Weinreich G. Age- and gender-dependent adherence with continuous positive airway pressure therapy. Sleep Med 2011; 12: 1034-1036

48 Ye L, Pien GW, Ratcliffe SJ et al. Gender differences in obstructive sleep apnea and treatment response to continuous positive airway pressure. J Clin Sleep Med 2009; 5: $512-518$

49 Mermigkis C, Bouloukaki I, Mermigkis D et al. CRP evolution pattern in CPAP-treated obstructive sleep apnea patients. Does gender play a role? Sleep Breath. Epub ahead of print 01. 09. 2011 